\title{
Boundary singularities of solutions of sublinear elliptic equations.
}

\author{
Philippe GRILLOT
}

\begin{abstract}
Let $\Omega$ be a domain of $\mathbb{R}^{N}, N \geq 3$, such that $0 \in \partial \Omega$. In this paper we study the behavior near 0 of any nonnegative solution $u \in C^{2}(\Omega) \cap C(\bar{\Omega} \backslash\{0\})$ of equation of the type $-\Delta u+a(x) u^{q}=0$ where $0<q<1$ and function $a$ behaves like a power of $|x|$.
\end{abstract}

\section{Introduction}

In this article we study the boundary behavior of the nonnegative solutions of sublinear elliptic equations of the type

$$
-\Delta u+a(x) u^{q}=0
$$

in a domain $\Omega$ of $\mathbb{R}^{N}, N \geq 3, q \in(0,1)$, with a possible isolated singularity at one point of the boundary. More precisely we assume that $0 \in \partial \Omega$ is the singular point and $a \in C^{1}(\Omega)$ with :

$$
\begin{aligned}
& a(x)=|x|^{\sigma}(1+o(1)) \\
& |\nabla a(x)|=O\left(|x|^{\sigma-1}\right)
\end{aligned}
$$

near 0 , where $\sigma$ is a given real.

Our first question is the following : let $u \in C^{2}(\Omega) \cap C(\bar{\Omega} \backslash\{0\})$ be a nonnegative solution of (1) in $\Omega$ such that

1991 Mathematics Subject Classification: 35J67.

Servicio Publicaciones Univ. Complutense. Madrid, 1999. 


$$
u=\phi \quad \text { on } \quad \partial \Omega \backslash\{0\}
$$

where $\phi$ is a given continuous function on $\partial \Omega$; can we extend $u$ as a continuous function defined in whole $\bar{\Omega}$ ? If not, the second point is to describe the precise behavior of $u$ near 0 .

This boundary singularity problem for sublinear elliptic equation is a new type of problem. In the superlinear case, the problem has been studied by analystic methods by Gmira and Véron [9] and Sheu [12] in the regular case, Fabbri and Véron [8] in the non regular case and by probabilistic methods by Le Gall [10] and Dynkin and Kuznetsov [7]. Recall that the singularity is removable only in the case $q>N+1 /(N-$ 1), when $\sigma=0$.

When the singular point lies in $\Omega$, equation (1) has been studied in the superlinear case $q>1$ in [6], [11] and [13] and in the sublinear case $q<1$ in [1] and [2].

In the present work, we consider the case where $\Omega$ is a ball, for example

$$
\Omega=B\left(x_{0}, \frac{1}{2}\right) \text { with } x_{0}=\frac{e_{N}}{2},
$$

where $\left(e_{1}, \ldots, e_{N}\right)$ is the canonical basis of $R^{N}$. Our results depend on the relative positions of $q, N$ and $\sigma$. The principal point is to obtain a priori estimates near 0 for the solutions of (1). In that aim, we first use two change of variables which lead us to a problem in the half space

$$
\mathbb{R}^{N+}=\left\{x \in \mathbb{R}^{N} / x_{N}>0\right\} \text {. }
$$

More precisely we introduce the following Kelvin transform:

$$
u(x)=\left|y+e_{N}\right|^{N-2} v(y) \quad \text { with } y+e_{N}=\frac{x}{|x|^{2}}
$$

where $e_{N}=(0, \ldots, 0,1) \in \mathbb{R}^{N}$. A straightforward computation implies that $v$ satisfies

$$
-\Delta v(y)+\left|y+e_{N}\right|^{(N-2) q-(N+2)_{a}}\left(\frac{y+e_{N}}{\left|y+e_{N}\right|^{2}}\right) v^{q}(y)=0
$$

for all $y \in \mathbb{R}^{N+}$. Remark that the singular point 0 is reduced to infinity by this transform. Now we use the classical Kelvin transform :

$$
v(y)=|z|^{N-2} w(z) \quad \text { with } \quad z=\frac{y}{|y|^{2}} .
$$


If $v$ satisfies (6) in $\mathbb{R}^{N+}$, then $w$ is solution of

$$
-\Delta w+b(z) w^{q}=0
$$

in $\mathbb{R}^{N+}$ with the singularity at 0 and where $b(z)=|z|^{(N-2) q-(N+2)} \mid y+$ $\left.e_{N}\right|^{(N-2) g-(N+2)} a\left(\frac{y+e_{N}}{\left|y+e_{N}\right|^{2}}\right)$. Because of $(2)$, we have

$$
b(z)=|z|^{\sigma}(1+o(1)) \text { near } 0 .
$$

Once we are reduced to an equation in $R^{N+}$, we make a new change of variables which leads us to an equation in the infinite cylinder $\mathcal{C}=$ $\mathbb{R} \times S_{+}^{N-1}$ where $S_{+}^{N-1}$ is the hemisphere of $S^{N-1}$ contained in $\mathbb{R}^{N+}$ : defining

$$
V(t, \theta)=|z|^{N-1} w(z)=r^{N-1} w(r, \theta)
$$

where $(r, \theta)$ are the spherical coordinate of $z$ and $t=-l n r$. Because of $(8), V$ satisfies :

$$
\left\{\begin{array}{l}
V_{t t}+N V_{t}+(N-1) V+\Delta_{S^{N-1}} V=g(t, \theta) V^{q} \text { in } \mathcal{C} \\
V(t, .)=\Psi(t, .) \text { on } \partial S_{+}^{N-1}=S^{N-2}
\end{array}\right.
$$

where $g$ is some nonnegative function in $\mathcal{C}$ and $\Psi(t,$.$) is some nonnegative$ function on $S^{N-2}$ with $\max _{S^{N-2}} \Psi(t,)=.O\left(e^{-(N-1) t}\right)$ when $t$ tends to $+\infty$.

In the first time, we give a priori estimate result. For this we introduce the first eigenfunction $\Phi_{1}$ of the Laplacian in $W_{0}^{1,2}\left(S_{+}^{N-1}\right)$ where $S_{+}^{N-1}$ is the hemisphere of $S^{N-1}$ contained in $\mathbb{R}^{N+}$. The function $\Phi_{1}$ is normalized by $\left\|\Phi_{1}\right\|_{\infty}=1$ and satisfies

$$
\left\{\begin{array}{l}
-\Delta_{S^{N-1}} \Phi_{1}=(N-1) \Phi_{1} \text { in } S_{+}^{N-1} \\
\Phi_{1}=0 \text { on } S^{N-2} .
\end{array}\right.
$$

Now the main point is to prove that any estimate of the mean value

$$
\bar{V}(t)=\int_{S_{+}^{N-1}} V(t, \theta) \Phi_{1}(\theta) d \theta
$$

implies an analogous estimates on $V$. Then we are reduced to give estimates on $\bar{V}$, wich reduces the problem to the resolution of ordinary 
differential inequalities. In that way we get a priori estimates for all nonnegative solution of (1) satisfying (4) for all continuous function $\phi$. Our main result concerning the priori estimates is the following.

Theorem 1. Assume $\phi$ is a continuous function on $\partial B$. Let $u \in$ $C^{2}(B) \cap C^{0}(\bar{B} \backslash\{0\})$ be any nonnegative solution of (1) satisfying (4). Then we have:

(i) If $q<\min \left(1, \frac{N+1+\sigma}{N-1}\right)$, then

$$
u(x)=O\left(|x|^{1-N}\right) \text { near } 0 .
$$

(ii) If $q>\frac{N+1+\sigma}{N-1}$, then

$$
u(x)=O\left(|x|^{\frac{2+\sigma}{1-q}}\right) \text { near } \quad 0 .
$$

(iii) If $q=\frac{N+1+o}{N-1}$, then

$$
u(x)=O\left(\left.|x|^{1-N}|\ln | x\right|^{\frac{1}{1-q}}\right) \quad \text { near } \quad 0 .
$$

Our results show that two effects one fighting each other, the nonlinear and the linear one, as it was the case in the interior problem [1], [2]. The nonlinear effect is governed by the possible existence of particular solutions of $(8)$ when $b(z)=|z|^{\sigma}$, given by :

$$
w^{*}(z)=C(N, q, \sigma)|z|^{\gamma} \quad \text { where } \quad \gamma=\frac{2+\sigma}{1-q} .
$$

The linear effect is governed by the solution of Poisson equation :

$$
\left\{\begin{array}{l}
-\Delta P=0 \text { in } \mathbb{R}^{N+} \\
P(0)=\delta_{0}
\end{array}\right.
$$

where $\delta_{0}$ is the Dirac mass at the origin. Recall that $P$ is given by $P(z)=P(r, \theta)=C_{N} r^{1-N} \Phi_{1}(\theta)$.

In a second part we prove more precise convergence results by using some techniques adapted to equations in an infinite cylinder, still used in $[11],[4],[3],[1]$.

Our main result is then the following : 
Theorem 2. Assume $\phi$ is a nonnegative continuous function on $\partial B$, identically equal to 0 in a neighborhood of 0 in $\partial B$. Let $u \in C^{2}(B) \cap$ $C^{0}(\bar{B} \backslash\{0\})$ be any nonnegative solution of (1) satisfying (4).

(i) Assume $q<1<\frac{N+1+\sigma}{N-1}$ (hence $2+\sigma>0$ ). Then, using Kelvin transforms (5) and (7), there exist $l \geq 0$ such that :

$$
\lim _{|r| \rightarrow 0}|r|^{N-1} w(r, \theta)=l \Phi_{1}(\theta) \quad \text { uniformly on } S_{+}^{N-1} \text {. }
$$

with $(r, \theta) \in \mathbb{R}_{+}^{*} \times S_{+}^{N-1}$ is the spherical coordinates of $z$ in $\mathbb{R}^{N+}$.

If $l=0$, then $u$ can be extended to a continuous function in $\bar{B}$. In that case $\bullet$ if $\sigma+1+q \leq 0$, then

$$
u(x)=O\left(|x|^{\gamma}\right) \quad \text { near } \quad 0
$$

with $\gamma=\frac{2+\sigma}{1-q}$. Using Kelvin transforms (5) and (7), the limit set in $C^{2}\left(S_{+}^{N-1}\right)$ of $r^{-\gamma} w(r,$.$) as r$ goes to 0 is contained in the set of nonnegative solutions of

$$
\left\{\begin{array}{l}
\Delta_{S^{N-1}} \omega+\gamma(\gamma-2+N) \omega-\omega^{q}=0 \text { in } S_{+}^{N-1} \\
\omega=0 \text { on } S^{N-2} .
\end{array}\right.
$$

- If $\sigma+1+q>0$, then there exists $k \geq 0$ such that

$$
\lim _{|r| \rightarrow 0}|r|^{-1} w(r, \theta)=k \Phi_{1}(\theta) \quad \text { uniformly on } S_{+}^{N-1} \text {. }
$$

Moreover, if $k=0$, then (20) holds and we have the same property as above.

(ii) Assume $q<\frac{N+\sigma+1}{N-1} \leq 1$ (hence $2+\sigma \leq 0$ ). Then (19) holds and if $l=0$, then $u \equiv 0$ near the origin.

(iii) Assume $\frac{N+\sigma+1}{N-1}<q<1$. Then as in (i) we have (20) and the inclusion property. Moreover, if $a(x)=|x|^{\sigma}$ and $\lim _{n \rightarrow+\infty} r_{n}^{-\gamma} w\left(r_{n},.\right)=$ 0 for some sequence $r_{n} \rightarrow 0$, then $u$ is identically equal to 0 near the origin.

Our paper is organized as follows :

1. Introduction 
2. Preliminary results

3. A priori estimates

4. Convergence results.

\section{Preliminary results}

Let $\mathrm{Cl}$ the infinity cylinder defined by $\mathcal{C l}=[1,+\infty) \times S_{+}^{N-1}$. For all function $V$ defined on $\mathcal{C l}$, we denote $\bar{V}$ the average of $V$ defined on $[1,+\infty)$ as in (13).

We start this section with some result which allows us to claim that a nonnegative solution $V$ of some elliptic equation in $\mathrm{Cl}$ is bounded as soon as its average $\bar{V}$ in $[1,+\infty[$ is.

Proposition 1. Let $\left(a_{1}, a_{2}, b_{1}, b_{2}, c_{1}\right) \in \mathbb{R} \times \mathbb{R}^{*} \times \mathbb{R}^{3}$. Assume that $g$ is a nonnegative bounded function on $C l$. Let $V \in C^{2}(C l) \cap C(\overline{C l})$ be any nonnegative solution of

$V_{t t}+\left(\frac{a_{1}}{t}+a_{2}\right) V_{t}+\frac{1}{t}\left(\frac{b_{1}}{t}+b_{2}\right) V+c_{1} V+\Delta_{S^{N-1}} V=g(t, \theta) V^{q}$ in $c l$

satisfying

$$
V=\Psi \text { on }[1,+\infty) \times S^{N-2}
$$

with $\Psi \in C\left([1,+\infty) \times S^{N-2}\right)$ be a nonnegative function and $\max _{S^{N-2}} \Psi(t,)=.O\left(e^{-\beta t}\right)$ for some $\beta>0$.

If $\bar{V}$ is bounded on $\left[1,+\infty\left[\right.\right.$, then $V$ belongs to $L^{\infty}(\mathcal{C l})$.

This proposition ensues from the two following lemmas. They are an adaptation of some result of [5] for a problem with the other sign in the cylinder, of the type

$$
\left\{\begin{array}{l}
W_{t t}+a_{0} W_{t}-l W+\Delta_{S^{N-1}} W+W^{Q}=0 \text { in } c l \\
W=0 \text { on }[1,+\infty) \times S^{N-2}
\end{array}\right.
$$

where $a_{0}, l$ are constants, with $l>0$, in the superlinear case $Q>1$.

Lemma 1. Under the assumptions of proposition 1 , for all $\gamma \in] 1, \frac{1}{1-q}[$, there exists $K=K(\gamma, N, q)>0$ such that for all $t \geq 2$ :

$$
\int_{t}^{t+1} \int_{S_{+}^{N-1}} 1 V \neq 0 \frac{|D V|^{2}}{V^{\beta}} \Phi_{1} d \theta d s \leq K
$$


where $\beta=2-\frac{1}{\gamma},|D V|^{2}=\left(V_{t}\right)^{2}+\left|\nabla_{S^{N-1}} V\right|^{2}$ and $1_{V \neq 0}$ denotes the caracteristic function of the set $\{(t, \theta) \in \mathcal{C l} / V(t, \theta) \neq 0\}$.

Proof. Since $V$ can vanish, we consider the function $U=V+\varepsilon$ for $\varepsilon \in(0,1)$. Because of $(23), U$ satisfies

$$
\begin{aligned}
U_{t t}+ & \left(\frac{a_{1}}{t}+a_{2}\right) U_{t}+c_{1} U+\Delta_{S^{N-1}} U+\frac{1}{t}\left(\frac{b_{1}}{t}+b_{2}\right) U \\
& \leq g(t, \theta) U^{q}+c_{1} \varepsilon+\frac{\varepsilon}{t}\left(\frac{b_{1}}{t}+b_{2}\right)
\end{aligned}
$$

in $\mathcal{C l}$. Now set $U=W^{\gamma}$, then $W \geq \varepsilon^{\frac{1}{\gamma}}$ in $\mathcal{C l}$ and from (26), $W$ satisfies in $\mathrm{Cl}$

$$
\begin{aligned}
W_{t t} & +\left(\frac{a_{1}}{t}+a_{2}\right) W_{t}+\Delta_{S^{N-1}} W+\frac{c_{1}}{\gamma} W \\
+ & \frac{1}{t \gamma}\left(\frac{b_{1}}{t}+b_{2}\right) W+\frac{\gamma-1}{W}\left(W_{t}^{2}+\left|\nabla_{S^{N-1}} W\right|^{2}\right) \\
& \leq \frac{C_{1}}{\gamma} W^{\gamma(q-1)+1}+\frac{c_{1}}{\gamma} \varepsilon^{\frac{1}{\gamma}}+\frac{\varepsilon^{\frac{1}{\gamma}}}{\gamma t}\left(\frac{b_{1}}{t}+b_{2}\right)
\end{aligned}
$$

where $C_{1}$ is a positive constant independant on $t$ and $\theta$. Multiplying (27) by $\Phi_{1}$ and integrating on $S_{+}^{N-1}$, the function $\bar{W}$ introduced in (13) satisfies

$$
\begin{gathered}
\bar{W}_{t t}+\left(\frac{a_{1}}{t}+a_{2}\right) \bar{W}_{t}+\left(\frac{c_{1}}{\gamma}-(N-1)\right) \bar{W} \\
+\frac{1}{\gamma t}\left(\frac{b_{1}}{t}+b_{2}\right) \bar{W}+\int_{S_{+}^{N-1}} A(t, \theta) d \theta \\
-\int_{S^{N-2}} \Psi \frac{\partial \Phi_{1}}{\partial \nu} d \theta \leq \frac{C_{1}}{\gamma} \int_{S_{+}^{N-1}}\left(W \Phi_{1}\right)^{j} d \theta+C_{2} \varepsilon^{\frac{1}{\gamma}}
\end{gathered}
$$

in $[1,+\infty)$, where $A(t, \theta)=\frac{\gamma-1}{W}\left(W_{t}^{2}+\left|\nabla_{S^{N-1}} W\right|^{2}\right) \Phi_{1}(\theta), j=\gamma(q-1)+$ $1 \in(0,1)$ and $C_{2}=\left(\int_{S_{+}^{N-1}} \Phi_{1} d \theta\right)\left[C_{1} \gamma^{-1}+\gamma^{-1} \max \left(0, \max _{t>1}\left(b_{1} t^{-2}+\right.\right.\right.$ $\left.\left.\left.b_{2} t^{-1}\right)\right)\right]$. Then from Jensen inequality and observing that $-\partial \Phi_{1} / \partial \nu \geq 0$ on $S^{N-2}$, we get :

$$
\bar{W}_{t t}+\left(\frac{a_{1}}{t}+a_{2}\right) \bar{W}_{t}+\int_{S_{+}^{N-1}} A(t, \theta) d \theta \leq \frac{C_{1}}{\gamma} \bar{W}^{j}+C_{2} \varepsilon^{\frac{1}{\gamma}}
$$




$$
-\left(\frac{c_{1}}{\gamma}-(N-1)\right) \bar{W}-\frac{1}{t \gamma}\left(\frac{b_{1}}{t}+b_{2}\right) \bar{W}
$$

in $[1,+\infty)$. On the other hand, Jensen inequality, the fact that $\Phi_{1}^{\gamma} \leq$ $\Phi_{1} \leq 1$ and that $\bar{V}$ is bounded imply that there exists $D>0$ such that for all $t \geq 1:(\bar{W}(t))^{\gamma} \leq \bar{U}(t) \leq D(1+\varepsilon)$. Therefore $\bar{W}$ is bounded on $[1,+\infty)$. From (29) we deduce that there exists $C_{3}>0$ such that

$$
0 \leq \int_{S_{+}^{N-1}} A(t, \theta) d \theta \leq C_{3}-\bar{W}_{t t}-\left(\frac{a_{1}}{t}+a_{2}\right) \bar{W}_{t}
$$

for all $t \geq 1$. Integrating twice (30) we obtain for all $t \geq 1$ :

$$
0 \leq \int_{t}^{t+1}\left(\int_{s}^{s+1}\left(\int_{S_{+}^{N-1}} A(\tau, \theta) d \theta\right) d \tau\right) d s \leq C_{4}
$$

where $C_{4}>0$ does not depend on $t$. Remark that for all nonnegative integrable function $f$, we have :

$$
\int_{t}^{t+1}\left(\int_{s}^{s+1} f(\tau)\right) d s \geq \int_{t+\frac{1}{2}}^{t+1}\left(\int_{t+1}^{s+1} f(\tau)\right) d s \geq \frac{1}{2} \int_{t+1}^{t+\frac{3}{2}} f(\tau) d \tau
$$

Hence we deduce from (31) :

$$
0 \leq \frac{1}{2} \int_{t+1}^{t+\frac{3}{2}} \int_{S_{+}^{N-1}} A(s, \theta) d \theta d s \leq C_{4} .
$$

Since $V=W^{\gamma}-\varepsilon,(32)$ implies for all $t \geq 2$ :

$$
0 \leq \int_{t}^{t+1} \int_{S_{+}^{N-1}} 1_{V \neq 0} \frac{|D V|^{2}}{(V+\varepsilon)^{\beta}} \Phi_{1} d \theta d s \leq C_{5}
$$

where $\beta=2-\gamma^{-1}$ and $C_{5}>0$ does not depend on $t$. Letting $\varepsilon$ tend to 0 in (33) we obtain (25) using Fatou lemma.

Lemma 2. Under the assumptions of proposition 1, for any $\varepsilon>0$ small enough there exists a positive constants $K_{1}$ such that for all $t \geq 2$ :

$$
\int_{t}^{t+1} \int_{S_{+}^{N-1}}(V(s, \theta))^{\frac{N}{N-1}-\varepsilon} d \theta d s \leq K_{1}
$$


Proof. Here we follow the ideas of the proof of [5] theorem 4.1. Let $\tau \in(0,1)$ be fixed. From [5] lemma 4.1, there exists a unique solution $\xi$ of problem

$$
\left\{\begin{aligned}
-\Delta_{S^{N-1}} \xi=\Phi_{1}^{-\tau} & \text { in } S_{+}^{N-1} \\
\xi=0 & \text { on } S^{N-2}
\end{aligned}\right.
$$

and there exists $K \geq 0$ such that $K^{-1} \Phi_{1} \leq \xi \leq K \Phi_{1}$ on $S_{+}^{N-1}$. Defining $Z(t)=\int_{S_{+}^{N-1}} V(t, \theta) \xi(\theta) d \theta$, we deduce from (23) that

$$
\begin{gathered}
Z_{t t}+\left(\frac{a_{1}}{t}+a_{2}\right) Z_{t}+\frac{1}{t}\left(\frac{b_{1}}{t}+b_{2}\right) Z+c_{1} Z-\int_{S^{N-2}} \Psi \frac{\partial \xi}{\partial \nu} d \theta= \\
\int_{S_{+}^{N-1}} V \Phi_{1}^{-\tau} d \theta+\int_{S_{+}^{N-1}} g V^{a} \xi d \theta
\end{gathered}
$$

hence from (24) there exists $A \geq 0$ such that

$$
\int_{S_{+}^{N-1}} V \Phi_{1}^{-\tau} d \theta \leq Z_{t t}+\left(\frac{a_{1}}{t}+a_{2}\right) Z_{t}+\frac{1}{t}\left(\frac{b_{1}}{t}+b_{2}\right) Z+c_{1} Z+A e^{-\beta t}
$$

On the other hand, since [5], there exist some constant $\mu$ and $\nu>0$ such that the function $\eta=\Phi_{1}\left(\mu-\nu \Phi_{1}^{1-\tau}\right)$ is a supersolution of (35). Since $\bar{V}$ is bounded:

$$
0 \leq Z(t) \leq \int_{S_{+}^{N-1}} V \Phi_{1}\left(\mu-\nu \Phi_{1}^{1-\tau}\right) d \theta<\infty
$$

Now integrating twice (36) between $t$ and $t+1$ for all $t \geq 2$ and using (37) we obtain after integrate by part the term $\left(a_{1} / t+a_{2}\right) Z_{t}$ :

$$
\int_{t}^{t+1}\left(\int_{a}^{s+1}\left(\int_{S_{+}^{N-1}} V \Phi_{1}^{-\tau} d \theta\right) d \tau\right) d s \leq D
$$

where $D>0$ does not depend on $t$. Then as in lemma 1 , we prove that there exists $K_{\tau}>0$ such that for any $t \geq 2$ :

$$
\int_{t}^{t+1} \int_{S_{+}^{N-1}} u \Phi_{1}^{-\tau} d \theta d \tau \leq K_{\tau}
$$

Then from estimates (25) and (38) and using Holder and Sobolev inequalities, we deduce (34) as in [5], lemma 4.1. 
We now give the proof of proposition 1 where the condition $q<1$ highly occurs.

Proof of proposition 1. In this proof, for $l \in \mathbb{N}^{*}, C_{i}$ denotes a positive constant independant on $t$. Set $f(t, \theta)=g(t, \theta) V^{q}-c_{1} V-\frac{1}{t}\left(\frac{b_{1}}{t}+b_{2}\right) V$. We know that $g$ is bounded on $\mathcal{C l}$ and because of (34), Young inequality implies that for all $t \geq 2$ :

$$
\|f\|_{L \frac{N}{N-1}-\varepsilon}\left([t-1, t+1] \times \overline{\left.S_{+}^{N-1}\right)} \leq C_{1} .\right.
$$

For all $j \geq 1$, define $K_{t}^{(j)}=\left[t-\frac{1}{j}, t+\frac{1}{j}\right] \times \overline{S_{+}^{N-1}}$. Because $V$ satisfies (23), Calderon-Zygmund theory ensures that for all $t \geq 2$ :

$$
\|V\|_{W^{2} \cdot \frac{N}{N-1}-\varepsilon\left(K_{t}^{(2)}\right)} \leq C_{2} .
$$

Then, since $\frac{N}{N-1}-\varepsilon<\frac{N}{2}$, Sobolev imbeddings imply :

$$
\|V\|_{L^{p_{1}\left(K_{t}^{(2)}\right)}} \leq C_{2}^{\prime}
$$

with $\frac{1}{p_{1}}=\frac{N-1}{N-\overline{\varepsilon(N-1)}}-\frac{2}{N}$. Using Calderon-Zygmund theory with some $p_{1}>\frac{N}{N-1}-\varepsilon$, we prove (40) with $p_{1}$ and $K_{t}^{(3)}$ respectively replacing by $\frac{N}{N-1}-\varepsilon$ and $K_{t}^{(2)}$.

Therefore Sobolev imbeddings imply :

$$
\begin{gathered}
\text { If } p_{1}>\frac{N}{2}, \text { then }\|V\|_{L^{\infty}\left(K_{t}^{(3)}\right)} \leq C_{3} . \\
\text { If } p_{1}=\frac{N}{2}, \text { then }\|V\|_{L^{p}\left(K_{\mathrm{t}}^{(3)}\right)} \leq C_{4} \quad \forall p \geq p_{1} .
\end{gathered}
$$

Applying another time Calderon-Zygmund theory with some $p>p_{1}$, we obtain (40) with $p$ and $K_{t}^{(4)}$ respectively replacing $\frac{N}{N-1}-\varepsilon$ and $K_{t}^{(2)}$ and we can use (42).

$$
\text { If } p_{1}<\frac{N}{2}, \text { then }\|V\|_{L^{p_{2}\left(K_{\mathrm{t}}^{(2)}\right)}} \leq C_{5}
$$

with $p_{2}$ such that $\frac{1}{p_{2}}=\frac{1}{p_{1}}-\frac{2}{N}$. Either $p_{2}>\frac{N}{2}$ and we are under the condition of 42), or we use (39) with $p_{2}>p_{1}$. We construct in that way a nondecreasing sequence $\left(p_{n}\right)$ such that $\frac{1}{p_{n}^{n}}=\frac{1}{p_{n-1}}-\frac{2}{N}$. Thus there exists 
$p_{n_{0}}>\frac{N}{2}$ and finally obtain the existence of some $j_{0} \geq 1$ and $C>0$ such that $\|V\|_{L^{\infty}\left(K_{0}^{(j 0)}\right)} \leq C$. This achieves the proof.

We end this section with the following convergence lemmas which will allow us to prove theorem 2 .

Lemma 3. Let $(A, \alpha) \in \mathbb{R}^{*} \times \mathbb{R}_{+}^{*}$. Consider a nonnegative Holder function $f$ in $C l$ satisfying :

$$
f(t, .)=O\left(e^{-\alpha t}\right) \text { uniformly in } S_{+}^{N-1}
$$

for large $t$. Let $Y \in C^{2}(\overline{\mathcal{C l}})$ be any nonnegative bounded solution of equation

$$
Y_{t t}+A Y_{t}+(N-1) Y+\Delta_{S^{N-1}} Y=f(t, \theta) Y^{q}
$$

in $\mathrm{Cl}$ and satisfying

$$
Y(t, .)=0 \quad \text { on } \partial S_{+}^{N-1}
$$

for all $t$. Then $Y_{t}$ and $Y_{t t}$ tends to 0 in $L^{2}\left(S_{+}^{N-1}\right)$ when $t$ tends to infinity and there exists $l \geq 0$ such that

$$
\lim _{t \rightarrow+\infty} Y(t, .)=l \Phi_{1} \quad \text { uniformly on } S_{+}^{N-1}
$$

Proof. Since $Y$ is bounded on $\overline{\mathrm{Cl}}$, Calderon-Zygmund theory, Sobolev imbedding and Schauder theory imply that there exists a constant $C>0$ such that

$$
\|Y\|_{C^{2, \beta q}(\overline{C l})} \leq C
$$

with $\beta \in] 0,1[$. Now define on the one hand the limit set

$$
\Gamma(Y)=\bigcap_{t \geq 1} \bigcup_{\tau \geq t} Y(\tau, .)^{C^{2}\left(S_{+}^{N-1}\right)} .
$$

As in [1], both $Y_{t}$ and $Y_{t t}$ tend to 0 in $L^{2}\left(S_{+}^{N-1}\right)$ when $t$ tends to infinity. Then $\Gamma(Y)$ is a connected compact subset of the set $E=$ $\left\{\omega \in C^{2}\left(S_{+}^{N-1}\right) /-\Delta_{S^{N-1}} \omega=(N-1) \omega\right.$ in $S_{+}^{N-1}, \omega \geq 0$ and $\omega=$ 0 on $\left.\partial S_{+}^{N-1}\right\}=\left\{l \Phi_{1} / l \in \mathbb{R}^{+}\right\}$. 
On the other hand multiplying (46) by $\Phi_{1}$, integrating on $S_{+}^{N-1}$ and using (45) and (49), we obtain

$$
0 \leq \bar{Y}_{t t}+A \bar{Y}_{t} \leq D e^{-\alpha t}
$$

for all $t \geq 1$ with $D>0$ and $\bar{Y}$ defined in (13). Because of (51), the function $\bar{G}: t \mapsto \bar{Y}_{t}+A \bar{Y}+\frac{D}{\alpha} e^{-\alpha t}$ is nonincreasing and lowerbounded on $\left[1,+\infty\right.$ (. Therefore there exists $\tilde{l} \in \mathbb{R}$ such that $\tilde{l}=\lim _{t \rightarrow+\infty} G(t)=$ $\lim _{t \rightarrow+\infty} A \bar{Y}(t)$ because $\bar{Y}_{t}$ tends to 0 in $L^{2}\left(S_{+}^{N-1}\right)$.

Finally, because of (49) and the fact that $\Gamma(Y)$ is included in $E$, there exists $l \in \mathbb{R}^{+}$and a sequence $\left(t_{n}\right)$ converging to infinity such that $Y\left(t_{n},.\right)$ tends to $l \Phi_{1}$ in $C^{2}\left(S_{+}^{N-1}\right)$ as $n$ tends to infinity. Thus we obtain $\tilde{l}=A l \int_{S_{+}^{N-1}} \phi_{1}^{2}(\theta) d \theta$. It would be the same for an other sequence and (48) holds.

In the same way, we can prove the analogous lemma:

Lemma 4. Let $(A, B, \alpha) \in \mathbb{R}^{*} \times \mathbb{R} \times \mathbb{R}_{+}^{*}$. Consider the Holder nonnegative function $f$ in $\mathrm{Cl}$ satisfying:

$$
|f(t, .)-1|=O\left(e^{-\alpha t}\right) \quad \text { uniformly on } S_{+}^{N-1} .
$$

Let $Y \in C^{2}(\overline{\mathcal{C l}})$ be any nonnegative bounded solution of equation

$$
Y_{t t}+A Y_{t}+B Y+\Delta_{S^{N-1}} Y=f(t, \theta) Y^{q}
$$

in $C l$ and satisfying (47) for all $t$. Then the limit set $\Gamma(Y)=\bigcap_{t \geq 1} \overline{U_{\tau \geq t}}$ $\overline{Y(\tau, .)} C^{2}\left(S_{+}^{N-1}\right)$ is a connected compact subset of the set $\{\omega \in$ $C^{2}\left(S_{+}^{N-1}\right) / \Delta_{S^{N-1}} \omega+B \omega-\omega^{q}=0$ on $S_{+}^{N-1}, \omega \geq 0$ and $\omega=$ 0 on $\left.\partial S_{+}^{N-1}\right\}$.

\section{A priori estimates}

In this section, we consider a nonnegative solution of equation (1) and give an a priori estimate near 0 of this solution.

Proof of theorem 1. Considering both changes of variables (5), (7) and (10), the function $V$ satisfies the equation (11) in the cylinder $\mathrm{Cl}=$ 
$[1,+\infty) \times S_{+}^{N-1}$, where $g$ is a nonnegative function in $\mathcal{C l}$, Holderian because of (3), satisfying because of (2):

$$
g(t, .)=O\left(e^{-\alpha t}\right) \text { uniformly on } S_{+}^{N-1}
$$

with $\alpha=N+1+\sigma-q(N-1)$. And

$$
V=\Psi \geq 0
$$

with $\Psi \in C(\mathrm{Cl})$ and satisfies for all $t \geq 1$ :

$$
\Psi(t, .)=O\left(e^{(1-N) t}\right) \text { uniformly on } \partial S_{+}^{N-1} \text {. }
$$

Now consider the function $\bar{V}$ defined in (13). Multiplying (11) by $\Phi_{1}$ and integrating on $S_{+}^{N-1}$, we obtain for all $t \geq 1$ :

$$
\bar{V}_{t t}+N \bar{V}_{t}-\int_{\partial S_{+}^{N-1}} V(t, \tau) \frac{\partial \Phi_{1}}{\partial \nu}(\tau) d \tau=\int_{S_{+}^{N-1}} g(t, \sigma) V^{q}(t, \sigma) \Phi_{1}(\sigma) d \sigma
$$

Since $\frac{\partial \Phi_{1}}{\partial \nu}$ is nonpositive on $\partial S_{+}^{N-1},(54),(56)$ and Jensen inequality imply that there exists $C>0$ such that for all $t \geq 1$

$$
\bar{V}_{t t}+N \bar{V}_{t} \leq C e^{-\alpha t} \bar{V}^{q} .
$$

We now distinguish three cases :

(i) $\alpha>0$ :

If $\bar{V}$ is not bounded, then it is nondecreasing on an interval $[T,+\infty)$ with $T>1$. Actually if $\bar{V}$ is not nondecreasing, there exists a sequence $\left(t_{n}\right)$ of strict maxima of $\bar{V}$ such that $t_{n} \rightarrow+\infty$ and $\bar{V}\left(t_{n}\right) \rightarrow+\infty$. Let $s_{n}$ be a real such that $\bar{V}\left(s_{n}\right)=\max _{\left[T, t_{n}\right]} \bar{V}(t)$, then we have $\bar{V}(t) \leq \bar{V}\left(s_{n}\right)$ for all $t \in\left[T, s_{n}\right]$. Integrate $(57)$ on $\left[T, s_{n}\right]$, we obtain

$$
\begin{aligned}
-\bar{V}_{t}(T)+N \bar{V}\left(s_{n}\right)-N \bar{V}(T) & \leq C \bar{V}^{q}\left(s_{n}\right) \int_{T}^{s_{n}} e^{-\alpha t} d t \\
& \leq \frac{C}{\alpha} \bar{V}^{q}\left(s_{n}\right) e^{-\alpha T} .
\end{aligned}
$$

As $\bar{V}\left(t_{n}\right) \leq \bar{V}\left(s_{n}\right), \bar{V}\left(t_{n}\right) \rightarrow+\infty$ and $q \in(0,1)$, we have a contradiction when $n$ tends to infinity in (58). 
Now we claim that $\bar{V}$ is bounded. Actually, if $\bar{V}$ is not bounded, $\bar{V}$ is nondecreasing on $\left[T,+\infty\left[\right.\right.$ and then $\lim _{t \rightarrow+\infty} \bar{V}(t)=+\infty$. On the other hand, because of (57), the function $G: t \mapsto \bar{V}_{t}(t)+N \bar{V}(t)-$ $C \int_{2}^{t} e^{-\alpha s} \bar{V}^{q}(s) d s$ is nonincreasing on $[T,+\infty)$. Therefore $G$ is bounded from above on $[T,+\infty)$ by a constant $D \in \mathbb{R}$. Morever $\bar{V}_{t} \geq 0$ on $[T,+\infty)$ and we deduce

$$
N \bar{V}^{1-q}(t) \leq D \bar{V}^{-q}(t)+C \alpha^{-1} e^{-\alpha T} \quad \text { for all } t \geq T .
$$

Then we obtain a contradiction as $t$ goes to infinity and $\bar{V}$ is bounded on $[T,+\infty)$. Then the assumptions of proposition 1 are achieved, with $a_{1}=0, a_{2}=N, b_{1}=b_{2}=0, c_{1}=N-1$ and $\beta=N-1$ in (24). Thus proposition 1 applies and $V \in L^{\infty}(\mathrm{Cl})$. Using changes of variables (10), (7) and (5), we obtain (14).

(ii) $\alpha<0$ :

If $\bar{V}$ is bounded, then we obtain (14) as above and since $\alpha<0$, that is $q>\frac{N+1+\sigma}{N-1}$, we have $|x|^{1-N}<<|x|^{\frac{2+\sigma}{1-q}}$ near 0 which implies (15).

If $\bar{V}$ is not bounded, then there exist $1<t_{0}<t_{1}$ such that $1<$ $\bar{V}\left(t_{0}\right)<\bar{V}\left(t_{1}\right)$. Let $e \in\left(t_{1},+\infty\right)$. We define

$$
s_{e}=\min \left\{s \in\left[t_{0}, e\right] / \max _{\left[t_{0}, c\right]} \bar{V}=\bar{V}(s)\right\} .
$$

Then $\bar{V}(t) \leq \bar{V}\left(s_{e}\right)$ for all $t \in\left[t_{0}, e\right]$. We claim that $\bar{V}_{t}\left(s_{e}\right) \geq 0$. Actually, if $\left.s_{e} \in\right] t_{0}, e\left[\right.$, then $\bar{V}_{t}\left(s_{e}\right)=0$. If $s_{e}=t_{0}$, then $\left.t_{1} \in\right] t_{0}, e\left[\right.$ implies $\bar{V}\left(t_{1}\right) \leq$ $\bar{V}\left(s_{e}\right)=\bar{V}\left(t_{0}\right)$ and this is false. If $s_{e}=e$, then $\bar{V}_{t}\left(s_{e}\right)<0$ would be a contradiction with $\bar{V}\left(s_{e}\right)=\max _{\left[t_{0}, e\right]} \bar{V}$.

Now integrate $(57)$ on $\left[t_{0}, s_{e}\right]$, we obtain since $\bar{V}_{t}\left(s_{e}\right) \geq 0$ :

$$
\begin{aligned}
N \bar{V}\left(s_{e}\right) & \leq C \int_{t_{0}}^{s_{e}} e^{-\alpha t} \bar{V}^{q}(t) d t+N \bar{V}\left(t_{0}\right)+\bar{V}_{t}\left(t_{0}\right) \\
& \leq C \bar{V}^{q}\left(s_{e}\right) \int_{t_{0}}^{s_{e}} e^{-\alpha t} d t+C_{0}
\end{aligned}
$$

where $C_{0}>0$ only depends on $t_{0}$. Therefore, because $\bar{V}\left(s_{e}\right) \geq \bar{V}\left(t_{0}\right)>1$, we have

$$
\bar{V}^{1-q}\left(s_{e}\right) \leq-\frac{C}{\alpha N} e^{-\alpha s_{e}}+C_{0}
$$


Since the function $r \mapsto-\frac{C}{\alpha N} e^{-\alpha r}+C_{0}$ is increasing, we deduce from $s_{e} \leq e, \bar{V}(e) \leq \bar{V}\left(s_{e}\right), q \in(0,1)$ and (59) that (59) holds for $e$ replacing $s_{e}$. Therefore there exist $D>0$ such that for all $t>t_{1}$ :

$$
\bar{V}(t) \leq D e^{-\frac{\alpha}{1-q} t}=D e^{-\left[N-1+\frac{2+\alpha}{1-q}\right] t} .
$$

Finally we introduce the function $U$ defined on $\mathrm{Cl}$ by

$$
U(t, \theta)=e^{\left[N-1+\frac{2+a}{1-q}\right] t} V(t, \theta)
$$

and its average $\bar{U}$ defined in (13). Because of $(60), \bar{U}$ is bounded on $\left(t_{1},+\infty\right)$ and $U$ satisfies (23) with $a_{1}=b_{1}=b_{2}=0, a_{2}=2-N-2 \gamma$ and $c_{1}=\gamma(\gamma+2-N)$ where $\gamma=\frac{2+\sigma}{1-q}$ and $\beta=-\frac{2+\sigma}{1-q}>0$ in (24) because $\alpha<0$. Moreover the assumptions of proposition 1 are achieved and then $U \in L^{\infty}(\mathrm{Cl})$. Using changes of variables (61), (10), (7) and (5), we obtain (15).

(iii) $\alpha=0$ :

If $\bar{V}$ is bounded, we use the fact that $|x|^{1-N}<<\left.|x|^{1-N}|\ln | x\right|^{\frac{1}{1-q}}$ near 0 and we obtain (16). If $\bar{V}$ is not bounded, then in the same way as above, we prove the following inequality which is similar to $(60)$ :

$$
\bar{V}(t) \leq D t^{\frac{1}{1-q}} .
$$

Finally, we use a function $W$ defined on $\mathcal{C l}$ by :

$$
W(t, \theta)=t^{\frac{1}{1-q}} V(t, \theta) .
$$

It satisfies (23) with $a_{1}=2 /(1-q), a_{2}=N, b_{1}=2 /(1-q)(2 /(1-q)-1)$, $b_{2}=N, c_{1}=N-1$ and $\beta=(N-1) / 2$ in (24) for example. Then the assumptions of proposition 1 are achieved, we still obtain (16).

\section{Convergence results}

In this last section, we prove theorem 2 . We distinguish two cases.

First case : we assume $q \leq \min \left(\frac{N+\sigma+1}{N-1}, 1\right)$.

Consider the function $V$ introduced in (10). Because of (11), $V$ satisfies (46) with $A=N$ and $f=g$. Moreover $V$ is bounded from 
theorem 1 on an set $C l=[2,+\infty) \times S_{+}^{N-1}$ and theorem 2 assumptions imply (47). Then lemma 3 ensures that (19) holds.

If $l=0$, then we introduce $\bar{V}$ defined in (13). Lemma 3 and $l=0$ imply $\lim _{t \rightarrow+\infty} \bar{V}(t)=\lim _{t \rightarrow+\infty} \bar{V}_{t}(t)=0$. On the other hand, because of (11), the function $\vec{V}_{t}+N \bar{V}$ is nondecreasing and then it is nonpositive in $[2,+\infty)$. Therefore the function $t \mapsto e^{N t} \bar{V}(t)$ is nonincreasing and then

$$
\bar{V}(t)=O\left(e^{-N t}\right) \quad \text { at } \quad \text { infinity } .
$$

(i) Assume $2+\sigma>0$.

If $\sigma+1+q \leq 0$, then we introduce the function $Y$ defined on $\mathcal{C l}$ by

$$
Y(t, .)=e^{(N-1) t} V(t, .)
$$

and we will prove that $Y(t,)=.O\left(e^{-\gamma t}\right)$ to obtain (20). Because of (11), $Y$ satisfies in $\mathrm{Cl}$ :

$$
Y_{t t}+(2-N) Y_{t}+\Delta_{S^{N-1}} Y=h(t, \theta) Y^{q}
$$

where from (2) there exists $C>0$ such that :

$$
h(t, \theta) \sim C e^{-(2+\sigma) t}
$$

near $+\infty$ and uniformly on $S_{+}^{N-1}$. The average $\bar{Y}$ of $Y$ satisfies in $[2,+\infty)$ :

$$
\bar{Y}_{t t}+(2-N) \bar{Y}_{t}-(N-1) \bar{Y}=\int_{S_{+}^{N-1}} h(t, \theta) Y^{q}(t, \theta) \Phi_{1}(\theta) d \theta
$$

We claim that $\bar{Y}$ is nonincreasing. Actually, if $\bar{Y}$ is not monotone, there exists a sequence $\left(t_{n}\right)$ of strict maxima of $\bar{Y}$ which tends to $+\infty$ and we have a contradiction from (66) and the fact $\bar{Y}\left(t_{n}\right)>0$ when we take (67) at large $t_{n}$. Because of $(63), \bar{Y}(t)$ tends to 0 at infinity and since it is nonnegative, we deduce that $\bar{Y}$ is nonincreasing in an interval $[T,+\infty)$ with $T \geq 2$. Now, from (66), there exists $K>0$ such that (67) implies in $[T,+\infty)$

$$
\bar{Y}_{t t}+(2-N) \bar{Y}_{t}-(N-1) \bar{Y} \leq K e^{-(2+\sigma) t} \bar{Y}^{q} .
$$


If we consider the function $E$ defined by

$$
E(t)=\frac{\bar{Y}_{t}^{2}}{2}-(N-1) \frac{\bar{Y}^{2}}{2}-K e^{-(2+\sigma) t} \frac{\bar{Y}^{q+1}}{q+1}
$$

then (68) ensures that $E$ is nondecreasing in $[T,+\infty)$. Therefore there exists $\tilde{l}=\lim _{t \rightarrow+\infty} E(t) \in \mathbb{R} \cup\{+\infty\}$. Since $\lim _{t \rightarrow+\infty} \bar{Y}(t)=0$, we deduce from (69) that $\lim _{t \rightarrow+\infty} \frac{\bar{Y}_{t}^{2}(t)}{2}=\tilde{l}$. Moreover $\bar{Y}$ is bounded and thus $\tilde{l}=0$. It implies that $E$ is nonpositive and we get

$$
\begin{aligned}
-\bar{Y}_{t} & \leq \bar{Y}^{\frac{q+1}{2}} e^{-\frac{(2+\sigma)}{2} t}\left[2 K+(N-1) e^{(\sigma+1+q) t}\right] \\
& \leq \bar{Y}^{\frac{q+1}{2}} e^{-\frac{(2+\sigma)}{2} t \tilde{K}}
\end{aligned}
$$

in $\left[T_{0},+\infty\right)$ with $T_{0} \geq T$ and $\dot{K}>0$. Without loss of generality, we can assume $\bar{Y}>0$ in $\left[T_{0},+\infty\right)$ and (70) implies that the function $\phi: t \mapsto-\bar{Y}^{-\frac{1-q}{2}}+\frac{2 \tilde{K}}{2+o} e^{-\frac{(2+\sigma)}{2} t}$ is nonincreasing in $\left[T_{0},+\infty\right)$. Since $\lim _{t \rightarrow+\infty} \phi(t)=0$, we deduce that $\phi$ is nonnegative and we obtain $\bar{Y}(t)=O\left(e^{-\gamma t}\right)$ near $+\infty$. Finally, using the function $U$ defined by $U(t, \theta)=e^{\gamma t} Y(t, \theta)$, its average and proposition 1 , we obtain $Y(t,)=$. $O\left(e^{-\gamma t}\right)$ which implies (20).

On the other hand, the assumptions of lemma 4 are fulfilled and we obtain the inclusion property of (i).

If $\sigma+1+q>0$, then we introduce the function $Z$ defined on $\mathcal{C l}$ by $Z(t, \theta)=e^{N t} V(t, \theta)$. Because of $(63), \bar{Z}$ is bounded and satisfies from (11)

$$
Z_{t t}-N Z_{t}+(N-1) Z+\Delta_{S^{N-1}} Z=h(t, \theta) Z^{q}
$$

in $\mathcal{C l}$ with $h(t, \theta) \sim e^{-(\sigma+1+q) t}$ near $+\infty$. Proposition 1 applies, $Z$ is bounded in $\mathcal{C l}$ and lemma 3 implies (22). If $k=0$, then we proceed as in case $\sigma+1+q<0$ : we introduce the function $E$ defined by $E(t)=\frac{1}{2} \bar{Z}_{t}^{2}(t)-e^{-(\sigma+1+q) t} \frac{\bar{Z}^{q+1}}{q+1}(t)$ to prove that $\bar{Z}(t)=O\left(e^{-\gamma t+t}\right)$ near $+\infty$ which implies (20) because of proposition 1 . We end this case as above.

(ii) Assume $2+\sigma \leq 0$. Then [1] ensures the result. 
Second case : we assume $\frac{N+\sigma+1}{N-1}<q<1$. From theorem 1, (20) holds and the proof of the end is similar to the one of first case.

Now assume $a(x)=|x|^{\sigma}$ and $\lim _{n \rightarrow \infty} r_{n}^{-\gamma} w\left(r_{n},.\right)=0$ for some sequence $r_{n} \rightarrow 0$, it remains to prove that $u \equiv 0$ near 0 . The function $U$ defined as above satisfies in $\mathrm{Cl}$

$$
U_{t t}+A U_{t}+B U+\Delta_{S^{N-1}} U=h(t, \theta) U^{q}
$$

where $A=2-N-2 \gamma<0, B=\gamma(\gamma+N-2)>0$ and $h$ is defined by

$$
h(t, \theta)=\left.\left.e^{-\beta t}|z+| z\right|^{2} e_{N}\right|^{-\beta}
$$

with $\beta=N+2-(N-2) q>0,(r, \theta)$ denotes the spherical coordinates of $z$ and $t=-\ln r$. We introduce the energy function $E$ defined in $[2,+\infty)$ by

$$
E(t)=\int_{S_{+}^{N-1}}\left(\frac{1}{2} U_{t}^{2}-\frac{1}{2}\left|\nabla_{S^{N-1}} U\right|^{2}+\frac{B}{2} U^{2}-\frac{1}{q+1} U^{q+1} h\right) d \theta
$$

We claim that $E$ is nondecreasing. Actually, because of (72), we have

$$
E^{\prime}(t)=-A \int_{S_{+}^{N-1}} U_{t}^{2} d \theta-\int_{S_{+}^{N-1}} \frac{1}{q+1} U^{q+1} h_{t} d \theta
$$

Denote by $e^{-t} \phi(\theta)$ the first coordinate of $z$ and remark that $\phi \geq 0$ on $S_{+}^{N-1}$. From $(73), h_{t}(t, \theta)=\beta e^{-\beta t}\left[e^{-2 t}+2 e^{-3 t} \phi(\theta)+e^{-4 t}\right]^{-\frac{\beta}{2}-1}\left[e^{-3 t} \phi(\theta)+\right.$ $\left.e^{-4 t}\right]>0$ and then, $E$ is nondecreasing. On the other hand, since there exists a sequence $r_{n} \rightarrow 0$ such that $\lim _{n \rightarrow+\infty} r_{n}^{-\gamma} w\left(r_{n},.\right)=0$, we deduce that $0 \in \Gamma(U)=\bigcap_{t \geq 2}{\overline{\mathrm{U}_{\tau \geq t} U(\tau, .)}}^{C^{2}\left(S_{+}^{N-1}\right)}$. Therefore, using the fact that $E$ is nondecreasing, we obtain as in [4] that $\Gamma(U)=\{0\}$. Thus, [1] implies that $u \equiv 0$ near 0 .

Aknowledgement- The author thanks M.-F. Bidaut-Véron for his suggestions during the preparation of this paper. 


\section{References}

[1] M.-F. BIDAUT-VERON and P. GRILLOT, Asymptotic behaviour of the solutions of sublinear elliptic equations with a potential, Applicable Anal., ( to appear).

[2] M.-F. BIDAUT-VERON, V. GALAKTIONOV, P. GRILLOT and L. VERON, Singularities for a semilinear equation with a nonLipschitz linearity in $\mathbb{R}^{2}$, J. Diff. Equ., ( to appear).

[3] M.-F. BIDAUT-VERON and T. RAOUX, Asymptotics of solutions of some nonlinear elliptic systems, Comm. Part. Diff. Eq.,21 (1996), 1035-1086.

[4] M.-F. BIDAUT-VERON and L. VERON, Nonlinear elliptic equations on compact Riemannian manifolds and asymptotics of Emden equations, Invent. Math. 106, 489-539 (1991).

[5] M. BOUHAR and L. VERON, Integral tepresentation of solutions of semilinear elliptic equations in cylinders and applications, Nonlinear Anal.23, 275-296 (1994).

[6] H. BREZIS and L. VERON, Remolvable singularities of some nonlinear equations, Arch. Rat. Mech. Anal. 75, 1-6 (1980).

[7] E. B. DYNKIN and S. E. KUZNETSOV, Superdiffusion and removable singularities for quasilinear P. D. E, Comm. Pure Appl. Math. 49, 125-176 (1995).

[8] J. FABBRI and L. VERON, Singular boundary value problems for nonlinear elliptic equations in non smooth domains, Adv. in Diff. Equ.( to appear).

[9] A. GMrRA and L. VERON, Boundary singularities of solutions of some nonlinear elliptic equations, Duke Math. J. 64, 271- 324 (1991).

[10] J. F. LE GALL, The brownian snake and solutions of $\Delta u=u^{2}$ in a domain, Prob. Theory Rel. Fields 102, 393-432 (1995).

[11] L. VERON, Singular solutions of some nonlinear elliptic equations, Nonlin. Anal. 5, 225-242 (1981). 
[12] Y. C. SHEU, Remolvable singularities for solutions of some nonlinear differential equations, Duke Math. J. 74, 701-711 (1994).

[13] C. YARUR, Singularidades de ecuaciones de Schrodinger estacionarias, Thesis doctoral, Univ. Complutense, Madrid (1984).

Laboratoire de Mathématiques et Physique Théorique CNRS UPRES-A 6083

Faculté des Sciences

Parc de Grandmont 37200 TOURS

FRANCE

Recibido: 11 de Noviembre de 1998 